P153 (continued)

were queried for weight-related eating behaviors (using Weight Related Eating Questionnaire) of routine restraint (RR), compensatory restraint (CR), susceptibility to external cues (SEC), and emotional eating (EE) in relation to CSWL (defined as having achieved a weight loss greater than $10 \%$ of starting weight).

Measureable Outcome and Analysis: Participants were dichotomized into those with CSWL $(n=973)$ and with no CSWL $(n=481)$ the relationship between CSWL (controlling for age and sex) as the dependent variable and weight-related eating behaviors as the independent variable was assessed using logistic regression (Stata/SE 14). Results: Those with CSWL have higher odds of having RR (OR: 1.3, p<0.05) and CR (OR: 1.1, p<0.05) and lower odds of having SEC (OR: 0.7, p<0.05) and EE (OR: 0.8, p<0.05) than those without CSWL.

Conclusions and Implications: Weight-related eating behaviors of participants in proprietary meal replacement weight-loss programs who have successfully lost weight differ compared to those who have not. Knowledge of the relationship between CSWL and weight-related eating behaviors can be used by coaches to assist participants in reinforcing those behaviors that support weight loss. These results are limited to participants who self-select for proprietary meal-replacement weight-loss programs and cannot be generalized to other weight-loss or maintenance programs.

Funding: Sanford Health - South Dakota State University Collaborative Research program; SD Board of Regents R\&D Innovation program

\section{P154 Self-Reported Young Adult Male Androgen Deficiency Correlation with Stress and Sleep}

Camille M. Charlier, BS, MOT, West Virginia University; Makenzie L. Barr, BS; Sarah E. Colby, PhD, RD, University of Tennessee, Knoxville; Melissa D. Olfert, DrPH, RDN, melissa.olfert@mail.wvu.edu, West Virginia University, 333 AG SC Building G28, Morgantown, WV 26506

Objective: Androgen deficiency in males has traditionally been predominantly limited to older men aged $50+$ years. However, the increase in the prevalence of chronic diseases in young males is expending the conventional scope of hypogonadism as emerging evidence suggests that the condition is positively correlated with obesity, diabetes and cardiovascular disease. Little is currently known of the relationships between hormonal disruption, stress and sleep in young adult males This study investigates lifestyle behavior patterns in male college students, including screening for androgen deficiency.

Study Design, Setting, Participants, Intervention: Over 1,100 college freshmen across 8 U.S. universities participated in the USDA-funded GetFruved study, a project aimed at identifying and improving lifestyle behaviors.
Outcome Measures and Analysis: A sub-sample of 386 male students was surveyed. Instruments used include the Androgen Deficiency in the Aging Male Questionnaire (ADAM) to assess androgen deficiency, the Perceived Stress Scale to measure stress levels and the Pittsburgh Sleep Quality Index (PSQI) to evaluate sleep quality. Logistic Regression Analysis of the data was completed using JMP and SAS software.

Results: One hundred twenty-eight male participants met the criteria for androgen deficiency as defined by the ADAM questionnaire. Univariate logistic regression showed statistically significant effects of increased stress $(\mathrm{p}<0.0001)$ and poor sleep $(\mathrm{p}<0.0001)$ on ADAM scores. Following categorization, sleep findings were confirmed by an agreement analysis $(\mathrm{p}<0.0001)$. When modeled together, stress showed a stronger effect than sleep on ADAM scores $(\mathrm{p}<0.0001$ versus $\mathrm{p}=0.1235$ respectively).

Conclusions and Implications: In young adult men, correlation was found between androgen deficiency and both increased stress levels and poor sleep quality, with stress displaying the strongest effect.

Funding: USDA

\section{P155 Using a Grocery List is Associated with Higher Diet Quality but not BMI in Parents of School-Aged Children}

Elizabeth H. Ruder, PhD, MPH, RD, ehrihst@rit.edu, Rochester Institute of Technology, 180 Lomb Memorial Drive, SLA (78) - 1693, Rochester, NY 14623;

Barbara A. Lohse, PhD, RD, Rochester Institute of Technology; Diane C. Mitchell, MS, RD, Pennsylvania State University; Leslie Cunningham-Sabo, PhD, RD, Colorado State University

Objective: Define the association between grocery list use, diet quality and weight status among parents of school-aged children.

Study Design, Setting, Participants, Intervention: Parents $(n=356)$ of children participating in Fuel for Fun, a school-based culinary and physical activity intervention, completed online, tested surveys on BMI, socio-demographics and eating behaviors. A subset $(n=83)$ completed ASA24 dietary assessment to obtain Healthy Eating Index (HEI).

Outcome Measures and Analysis: Participants who shopped with a grocery list "most of the time" or "almost always" were dichotomized versus those responding "sometimes/seldom/do not do". Chi-square and independent samples t-tests compared differences in socio-demographics, eating behavior, HEI and BMI by grocery list use. Linear regression models measured the association between shopping with a list and HEI or BMI controlling for covariates.

Results: Parents (mean age $38.0 \pm 6.5$ y) were mostly white (90\%), female (87\%), and highly educated. BMI was overweight/obese for $42 \%$. Total HEI (scored 0-100) ranged from 22.0 - 77.4, mean $55.4 \pm 12.4 \mathrm{SD}$; grocery 